Books, videos, CD-ROMs, DVDs and any

other relevant items submitted for a review

in the $B D J$ should be addressed to: Kate

Maynard, Assistant Editor, British Dental

Journal, NPG, 4-6 Crinan Street, London,

N1 9XW

\section{D head and neck anatomy with special senses and basic neuroanatomy (DVD-ROM)}

\author{
B. Berkovitz, C. Kirsch, B. J. Moxham, \\ G. Alusi, T. Cheeseman \\ UK: Primal Pictures Ltd \\ price $€ 159.00$ \\ ISBN 9781904369691
}

Anyone tempted to pay out a lot of money has a right to expect a lot of anatomy and that is what this DVD contains. Using computerised reconstructions from slice dissections, several views of the head and neck, brain and special senses are available that can be rotated through $360^{\circ}$ or stripped layer by layer. Click the pointer over any structure and a description in traditional anatomy textbook style appears at the side of the image.

There are some serious errors; the TMJ articular cartilages are labelled as hyaline cartilage and the oral cavity is lined by respiratory mucosa to name just a couple of screamers I came across. The nerves and vasculature are shown in high detail - some of the nerve views look like a bowl of vermicelli tipped over a skull and are extremely difficult to unravel. An editorial deletion of some of the minor branches would make these views much more useful. The main limitation to these views is the lack of internal detail of the mouth, nose or larynx.

MRI scans can be followed sequentially in all three planes accompanied by an anatomical reconstruction. A selection of slides includes anatomy illustrations (textbook derived), clinical topics (a few gory pictures), MRI and absolutely stunning dissections. Each dissection is accompanied by a list of structures; a mouse click highlights the structure. Unfortunately the labels are not linked to the descriptions accompanying the reconstructions. There are also some movie clips, mainly of muscle actions. Search facilities enable the user to find structures in any part of the package and hyperlinks in the descriptions lead to other pictures of the same thing. There are quizzes in either 'label or identify' or 'true-false' format. The MCQ section has many ambiguous questions and many questions in both sections are anatomical trivial pursuits with a vengeance. They really do need a very heavy editorial hand to get the questions in proportion and worded unambiguously.

For the price of this DVD you could buy four or five anatomy textbooks or atlases, so is it value for money? Most textbooks will give added value not found on the DVD in the form of description of complex functions such as mastication or swallowing, an overview of structures as well as clinical relevance, which is lacking in this DVD. These days, many textbooks have CDs or access to publishers' websites for additional pictures. The price is not right for students and even for MFDS candidates a textbook and atlas would be better value until Primal have refined their product considerably.

M. Atkinson

\section{Insights, facts and stories behind Trigeminal Neuralgia}

J. M. Zakrzewska

Trigeminal Neuralgia Association price $€ 16.50$ inc p\&tp, pp 403 ISBN 0967239346

This book is a timely addition to texts on facial pain, written by a well-known author in the field of Trigeminal Neuralgia (TN). In particular, it is the first text to bring together the key themes of orofacial pain in an accessible form for patients and undergraduate dental students. The text is divided into 12 chapters that follow each other in a logical clinical fashion. Chapter 1 covers the presentation and diagnosis of TN; chapters 2 and 3 outline the fundamental anatomical principles and epidemiology of TN. Chapter 4 deals with the investigations and chapter 5 evaluates how best to optimise patient care. Chapters 6 and 7 discuss the medical and alternative methods of managing TN. Chapters 8 and 9 address the decision-making strategies and surgical management for TN. Chapter 10 addresses the recurrence and complications and chapters 11 and 12 discuss the importance of support groups and relevant research questions.

This book shows the advances in evidence based care for TN patients that have led to a greater understanding and improved management of TN. Furthermore, the text also explores novel methods used in the diagnosis and possible management strategies for TN. The text reads well with each chapter starting with a wonderful medical illustration by Malcolm Willett and the text is often 'dotted' with personal statements, poems or illustrations by patients that bring the text alive. The author also makes good use of figures and tables are used throughout and they all provide useful summaries with regard to pain history, differential diagnosis, treatment goals, epidemiology, clinical study design and evaluation and evidence base for management of TN. They are designed to help students take a questioning view of a topic, and perhaps to realise the complexity of diagnosis and management of TN and related conditions. Furthermore, the contents will certainly be useful to provide patients, undergraduates and postgraduates with a realistic overview of the subject and would be an essential 'tome' for university libraries.

Personally, I would wholeheartedly recommend the book to all TN patients, dental and medical undergraduates, and those post-qualification practitioners (specialising or not) who feel the need for an introduction to Trigeminal Neuralgia.

T. Renton 

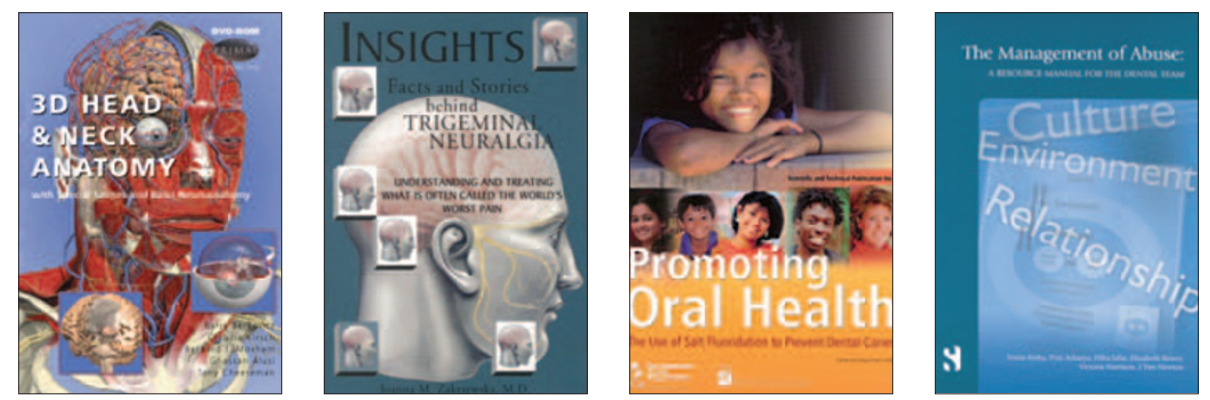

\section{Promoting oral health: the use of salt fluoridation to prevent dental caries}

\author{
S. Estupiñán-Day \\ USA: Pan American Health Organization \\ price $\$ 28.00$, pp 128 \\ ISBN 9275116156
}

This publication is the result of a collaboration between the Pan American Health Organization (PAHO) and the Kellogg Foundation. It is a specialised text and appears to be aimed at policy makers and health planners working at a national level.

The publication is comprised of 111 pages divided into 10 chapters. There are three distinct sections encompassing:

1. The history of salt fluoridation

2. The planning, launching, running, monitoring and evaluation of salt fluoridation programmes

3. Recommendations for setting up and operating a successful salt fluoridation programme, legal issues and standardised research protocols.

The book is best described as reference material for those involved in proposals for, and implementation of, salt fluoridation programmes. There is a detailed table of contents, but no index, with the result that it can be difficult to locate specific information. The nature of the subject matter is quite specialist in nature; however, the author has endeavoured to include tables and diagrams wherever possible to clarify the points made.

This is a detailed overview of implementation of salt fluoridation programmes, and would be a useful reference tool for those with a special interest in this field. It would perhaps have benefited from the inclusion of additional sections comprising the advantages and disadvantages of salt fluoridation programmes when compared to other oral health measures, and potential problems and possible solutions which may be encountered when initiating salt fluoridation programmes.

Overall, there are probably more suitable texts for the general dental population wishing to expand their knowledge regarding oral health promotion and salt fluoridation. For those, however, with a special interest in this subject, or those who are actively involved in oral health promotion and fluoridation at a regional or national level, this could make for interesting reading.

L. Molyneux and A. J. Preston

\section{The management of abuse: a resource manual for the dental team}

\begin{tabular}{l}
\hline J. T. Newton, E. Bower \\
UK: Stephen Hancocks Ltd (www.shancocksItd.com) \\
price Ł29.95, pp 112 \\
ISBN 945614534
\end{tabular}

This book tackles a difficult but necessary issue. I was surprised to find it is the only book presently to examine abuse from a dental perspective. The book is primarily aimed at general practice. This is an underrepresented area in dentistry however, and I suspect its appeal will be far wider. This book can be read from start to finish or it can also be used as a reference for the whole dental team.

The introductory chapter defines terminology and the evolution of current legislation. This was the hardest part of the book to read. The legal framework is essential for greater understanding of the issue, but it may have been easier to read if it was from a dental perspective - on common ground with its reader. The layout for the rest of the book, in contrast, is excellent.
The following three chapters are devoted in turn to different classification of abuse: child, domestic and vulnerable adults. The structure of each chapter follows a similar pattern - background, definitions, prevalence, risk factors, signs of abuse, advice for the dental team, specific legislation, further developments and reading. In particular it addresses the role of the GDP in cases of suspected abuse and the importance of appropriate multi-agency communication. There is an inevitable overlap between chapters but this serves to reinforce important issues. These chapters deliver valuable information in an easy to read manner with succinct flowcharts, diagrams and photos.

There is a useful PDF file available on the publisher's website for legislation developments since publication. The book states all legislation, policy and procedures are applicable to England only. It advises GDPs outside England that principles will be similar but to contact individual Health Departments for further detail. A brief description of differing legislation pertaining to each country could perhaps be useful through the PDF file.

For a fee, verifiable CPD can also be accessed through the publisher's website.

The final chapter links all aspects of prevention and detection of abuse in practice. There is plenty of practical advice from recruitment of staff to guidelines in promotion of a caring environment.

From diagrams for recording injuries to examples of questions to ask suspected victims of abuse, the appendices are an invaluable aspect of this book.

This book effectively and concisely bridges the gap between general advice for health professionals and the dental literature focused on signs and symptoms of abuse. Overall I would recommend it as an essential part of a practice library.

F. Watson 